\title{
Development of a method for classifying and transmitting high-resolution feeding behavior of fish using an acceleration pinger
}

\author{
J. Horie ${ }^{2,6^{*}}$, H. Mitamura ${ }^{1,3}$, Y. Ina ${ }^{1}$, Y. Mashino ${ }^{1}$, T. Noda ${ }^{4}$, K. Moriya' ${ }^{1}$ N. Arai ${ }^{3,5}$ and T. Sasakura ${ }^{2}$
}

\begin{abstract}
Background: Monitoring the feeding behavior of animals in the wild is key to understanding their energetics and the influence of the environment on their survival. Recently, a novel acceleration transmitter that processes acceleration data onboard and outputs identification results has been developed by AquaSound Inc. (Kobe, Japan) to investigate feeding biology in fish. To date, few attempts have been made to identify the feeding behavior of fish using transmitters, and none of these attempts accomplished classification of alternative feeding behaviors according to prey items. The objective of this study was to develop an algorithm that can be incorporated in the acceleration transmitter and can identify alternative feeding behaviors in fish, using red-spotted grouper (Epinephelus akaara) as a model species.
\end{abstract}

Results: Most of the identification algorithms describing feeding behavior in fish developed in previous studies used a combination of acceleration and angular velocity. In this study, we constructed an algorithm based on three-axis accelerometry data alone, since a gyroscope consumes much more electricity and would shorten the battery life of the transmitter. Acceleration data were obtained in tank experiments. Feeding behaviors, induced by feeding three types of live prey (Trachurus japonicus, Metapenaeus ensis and Hemigrapsus sanguineus), as well as other behaviors (routine and escape movements), were simultaneously recorded at $200 \mathrm{~Hz}$ by acceleration data loggers, implanted in the abdominal cavities of fish, and by a video camera. A decision tree, including a three-dimensional lookup table, was constructed to classify the behaviors into four behavior classes: shrimp-eating, fish-eating, crab-eating and other behaviors. The classification accuracy was estimated to be 0.77 ( $F$-measure) for shrimp-eating, 0.73 for fish-eating, 0.71 for crab-eating and 0.78 for other movements, using fivefold cross-validation.

Conclusions: The algorithm developed in this study could be incorporated into the transmitter, which would record acceleration data at high frequency $(200 \mathrm{~Hz})$, process the data onboard and output classification results of behaviors. This method would reveal more aspects of fish biology, such as individual feeding strategies.

Keywords: Biotelemetry, Acceleration transmitter, Lookup table

\section{Background}

One of the key goals in fish biology is to understand feeding behavior. When feeding, most fish exhibit characteristic changes in acceleration, which are, in most cases, distinct from those of routine movements [1]. Feeding behavior can thus be investigated in detail by measuring

\footnotetext{
*Correspondence: horie@aqua-sound.com

${ }^{6}$ Present Address: Graduate School of Informatics, Kyoto University,

Yoshida-honmachi, Sakyo-ku, Kyoto, Kyoto 606-8501, Japan

Full list of author information is available at the end of the article
}

characteristic changes in acceleration. Instruments that are widely used to measure physical variables in/around target fish in the wild are animal-borne data loggers and ultrasonic transmitters (aka pingers or acoustic transmitters). Previous studies have shown that animal-borne acceleration and/or gyroscope data loggers are useful to analyze the feeding behaviors of various species of fish [2-4]. However, a data logger has the disadvantage that it must be retrieved after attachment to a target fish (to download the data); this is often extremely difficult, 
depending on the species. In contrast, a pinger does not have this disadvantage; data are transmitted ultrasonically. In fact, pingers have been widely used for remotely measuring physiological and behavioral variables of freeliving animals [5]. However, the amount of information that a pinger can send per unit of time is critically limited, since underwater communication is compromised by multipath propagation and strong signal attenuation. It follows that the sampling frequency of acceleration afforded by a pinger tends to be much lower (by $5-10 \mathrm{~Hz}$ ) than that of a data logger. Sampling frequency can present a major problem, according to Broell et al. [2], who suggested that the sampling frequency should be more than $100 \mathrm{~Hz}$ for reliable identification of the feeding behaviors of predatory fish.

Nevertheless, a few novel acceleration pingers have been developed successfully to analyze the biological features of various fish. One of the best-known acceleration pingers is the V9AP (Vemco Inc., Nova Scotia, Canada; sampling frequency: 5 or $10 \mathrm{~Hz}$ ), which transmits general variables such as a root mean square (RMS) calculated from three-axis acceleration. The outstanding advantage of such a method is that the pinger can detect the movements of a target animal using relatively simple statistics. These pingers have been used to study the field activity and metabolic rates of bonefish [6] and giant Australian cuttlefish [7], the spatial ecology and residency patterns of the great barracuda [8], the seasonal residency of juvenile sand tiger sharks [9], energy use by wild adult Pacific salmon [10], and seasonal changes in the activity levels of horseshoe crabs [11]. Another well-known acceleration pinger is the AccelTag (Thelma Biotel, Trondheim, Norway; sampling frequency: $20 \mathrm{~Hz}$ ). This is a customadapted pinger that transmits behavior-specific parameters derived from three-axis acceleration data such as tilt angle, roll angle, lateral acceleration and vertical acceleration. This method, in which a pinger transmits not raw acceleration data but several simplified parameters derived onboard from enormous amounts of data, has resolved the essential problem of pingers, i.e., the inability to transmit large amounts of data. In fact, the AccelTag has been utilized to detect specific behaviors in fish, such as burrowing movements of Lusitanian toadfish [12, 13] and the tail beat activity of red-spotted grouper [14]. Despite these advances, pinger-mediated remote measurements of feeding behavior in fish remain challenging; the sampling frequency is too low $(<100 \mathrm{~Hz})$ to allow accurate classification of feeding behavior.

Recently, we have developed a new acceleration pinger (AquaSound Inc., Kobe, Japan) that samples acceleration data at a high frequency $(200 \mathrm{~Hz})$, classifies the behaviors of target fish via onboard processing and outputs the results of such classifications. The pinger must initially be programmed with a classification algorithm based on three-axis acceleration data. Such algorithms should not be elaborate; long processing times may compromise the pinger's other operations (sampling and transmitting). In this study, we developed a decision tree, including a three-dimensional lookup table that serves as the classification algorithm. The major advantage of this approach is that several key parameters are examined in detail. A lookup table alone requires a great deal of program memory. The combination of a lookup table and a decision tree enhances classification accuracy within the extent that the algorithm does not consume excessive memory. We first conducted tank experiments using acceleration data loggers to obtain acceleration data. We chose redspotted groupers (Epinephelus akaara) as the model species. This fish exhibits strong site fidelity [15] and is a typical sit-and-wait predator that remains still until food items are found (triggering feeding behavior) or a threat is detected (triggering escape behavior). We assumed that the foraging tactics of this fish were relatively advantageous for us in developing the algorithm; this species does not frequently perform dynamic behaviors, other than while feeding. Thus, feeding behavior was expected to be distinguishable from other behaviors.

\section{Methods}

\section{Study animals}

Eighteen mature red-spotted groupers [total length (TL): $35.0 \pm 3.7 \mathrm{~mm}$; body mass (BM): $687 \pm 197 \mathrm{~g}$ ] obtained from local fish dealers (in Osaka and Maizuru, Japan) were used. Red-spotted groupers grow up to about $60 \mathrm{~cm}$ in length and are found around the coasts of southern Honshu (Japan) and eastern China [16]. Previous studies of stomach contents have shown that mature fish feed principally on smaller fish, shrimps and crabs [15].

\section{Tank experiments}

Tank experiments using data loggers were conducted in October-November 2013, October-December 2014 and October-December 2015 at Maizuru Fisheries Research Station, Kyoto University, Kyoto, Japan. Eighteen red-spotted groupers were individually kept in fiberreinforced plastic (FRP) tanks (length: $70 \mathrm{~cm}$; width: $100 \mathrm{~cm}$ ) in which seawater circulated at a temperature of $17.2 \pm 1.1^{\circ} \mathrm{C}$ to a depth of $70 \mathrm{~cm}$. An acceleration data logger (size: $60 \times 6 \times 12 \mathrm{~mm}^{3}$; mass: $7 \mathrm{~g}$ in air; measurement range: $\pm 16 \mathrm{~g}$; sampling frequency: $200 \mathrm{~Hz}$; Biologging Solutions Inc., Tokyo, Japan) was surgically implanted into the abdominal cavity of each grouper. Surgical treatments were carried out under anesthesia induced by $0.05 \%$ phenoxyethanol. An incision approximately $20 \mathrm{~mm}$ in length was made in the abdomen (several centimeters above the vent) of the fish, and the logger 
was inserted. The wound was closed using an operating needle and sutures. The implant operation took approximately $5 \mathrm{~min}$. The weight of the logger in water was less than $3 \%$ of the weight of the grouper. After surgery, the groupers were acclimatized for at least 1 week. During the acclimatization period, we supplied the groupers with three types of live prey: fish (Japanese horse mackerel, Trachurus japonicus), shrimps (Metapenaeus ensis) and crabs (Hemigrapsus sanguineus). Subsequently, feeding behaviors were logged as three-axis acceleration data using the data loggers and simultaneously recorded as video images using a video camera (HDR-CX720V; SONY, Tokyo, Japan). The video images were used to associate all acceleration data with each of three behavioral patterns (fish-eating, shrimp-eating, or crab-eating). The data loggers were set to record fish behaviors for 25 min each day for 5-10 days. Shrimp-eating and crabeating events were sampled between 15:00 and 18:00, while fish-eating events were sampled between 18:00 and 21:00. These time frames were chosen based on a study of the feeding habits of red-spotted grouper [17].

\section{Data analyses}

Data analyses were performed using MATLAB (MathWorks, Natick, MA, USA). We first extracted all behavioral events from the three-axis acceleration datasets. An 'event' was defined as the $0-250-\mathrm{ms}$ interval commencing at the time when acceleration on any axis surpassed the threshold $(2.0 \mathrm{~g})$. This threshold was chosen to ensure that we detected all feeding events but not tiny, subtle routine movements.

The acceleration along each axis $\left(a_{x}, a_{y}\right.$ and $\left.a_{z}\right)$ was relabeled because an instrument inserted into the abdominal cavity of a fish would not be completely fixed. In the tank experiments, the $y$-axis was directed forward, but $x$ - and $z$-axes were not consistently directed vertically and laterally, judging from acceleration data from six red-spotted groupers over 10 days in which they did not move. Therefore, we termed the acceleration along the longitudinal axis (body axis $a_{b}$ ) $a_{y}$ and acceleration in the orthogonal direction $\left(a_{r}\right) \sqrt{a_{x}^{2}+a_{z}^{2}}$ (Fig. 1). The vectorial sum of all acceleration axes was thus $|a|=\sqrt{a_{x}^{2}+a_{y}^{2}+a_{z}^{2}}$.

\section{Results}

\section{Acceleration data}

In the tank experiments, we observed 49 shrimp-eating events ( $n=5$ fish), 9 fish-eating events ( $n=3$ fish), 15 crab-eating events ( $n=3$ fish) and 45 other behavioral events $(n=8$ fish). More details about the feeding events are given in Table 1.

A typical example of each behavior is shown in Fig. 2. At first glance, it seemed that (a) most of the

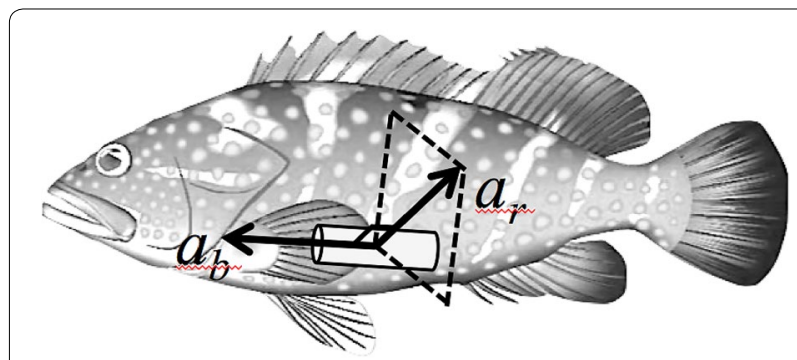

Fig. 1 Directions of acceleration (longitudinally and orthogonally)

characteristic changes in acceleration ended within $50 \mathrm{~ms}$ after event initiation, (b) the amplitude and duration of acceleration during fish-eating and other (escape) behavior tended to be much greater than those during any other behaviors, while (c) acceleration during crabeating and other (routine) behavior was lower than that during shrimp-eating, and (d) the duration of other behaviors (routine and escape) was exceptionally long in certain cases. We hypothesized that these characteristics were key components when selecting parameters to classify feeding behaviors.

\section{Classification phase I}

We first constructed a lookup table to classify feeding behaviors. For accurate classification, parameters used in a lookup table not only need to be characteristic of each type of behavior, but also somewhat independent of each other. In terms of the characteristics mentioned above, the amplitude and duration of acceleration are good examples of such parameters. Thus, we first divided each event into three phases using the border times $t_{S}$ and $t_{E}$ : the commencement phase ( 0 to $\left.t_{s} \mathrm{~ms}\right)$; the transition phase ( $t_{s}$ to $\left.t_{E} \mathrm{~ms}\right)$; and the final phase ( $t_{E}$ to $250 \mathrm{~ms}$ ). The commencement phase would describe the intensity of a movement; the final phase would reflect whether a fish became completely stationary or not; and the transition phase would support the other two parameters. Parameters from each of the three phases would be independent. We chose the standard deviation of $a_{b}$ in the commencement phase as parameter I; the standard deviation of $a_{r}$ in the transition phase as parameter II; and the standard deviation of $|a|$ in the final phase as parameter III. We assumed that $a_{b}$ was the most significant axis early in the behavior and that $|a|$ reflected even minimal movement along any axis. The border times $t_{S}$ and $t_{E}$ were chosen to be 55 and $120 \mathrm{~ms}$, respectively, based on the data of Fig. 3, where the maximum classification accuracy (defined below) was evident. Finally, the distribution map was created, as shown in Fig. 4.

Based on the distribution (Fig. 4), we estimated the statistical population using a three-dimensional Gaussian 
Table 1 Number of feeding events per individual that performed the behaviors

\begin{tabular}{llcl}
\hline Indiv. no. & Crab-eating & Shrimp-eating & Fish-eating \\
\hline 1 & 3 & 3 & 5 \\
2 & 0 & 11 & 0 \\
7 & 0 & 0 & 3 \\
12 & 0 & 11 & 0 \\
14 & 0 & 11 & 0 \\
15 & 8 & 13 & 0 \\
17 & 4 & 0 & 0 \\
18 & 0 & 0 & 1 \\
\hline
\end{tabular}

distribution (Fig. 5). The probability density of each type of behavior was calculated. Other (routine) behavior was condensed into very narrow ranges of all three parameters. Fish-eating spreads toward the directions of parameters I and II, but was noticeably limited in the direction of parameter III. Shrimp-eating was between other (routine) and fish-eating behavior. Crab-eating overlapped somewhat with shrimp-eating and other (routine) behavior. Other (escape) behavior spanned almost the entire distribution, although the density thereof was rather low.

The classification algorithm, i.e., the lookup table, was derived from the probability density distribution. First, the three-dimensional volume was divided into cells. The

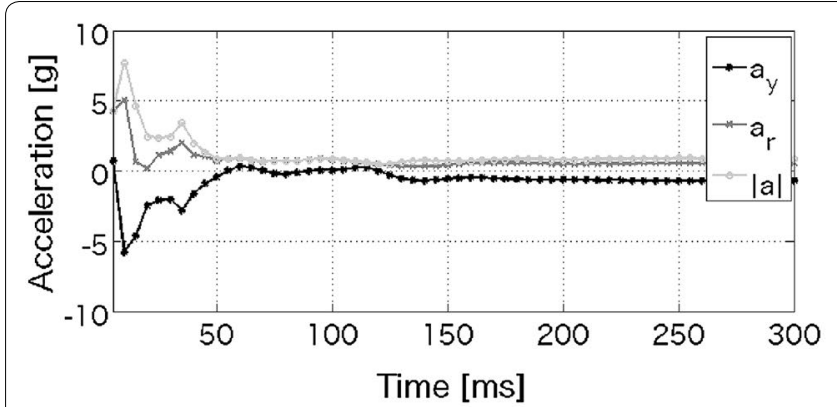

a

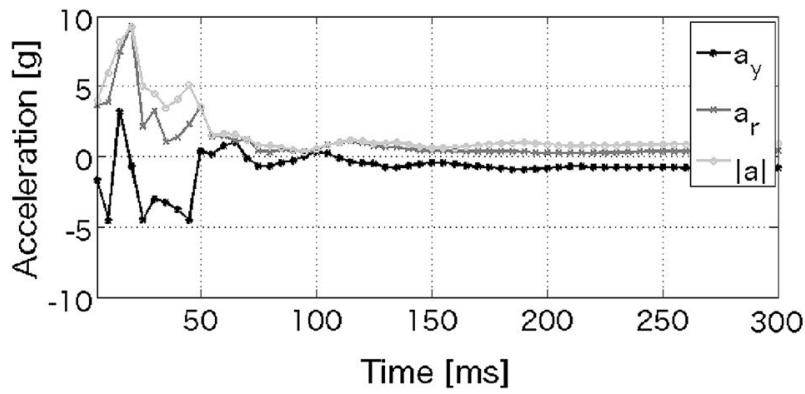

b

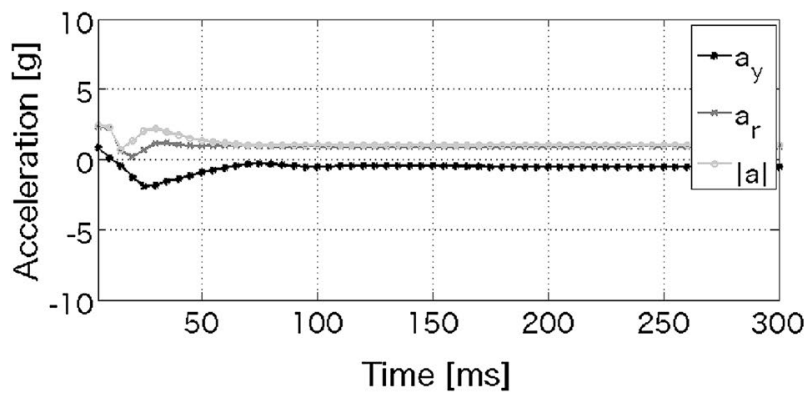

C

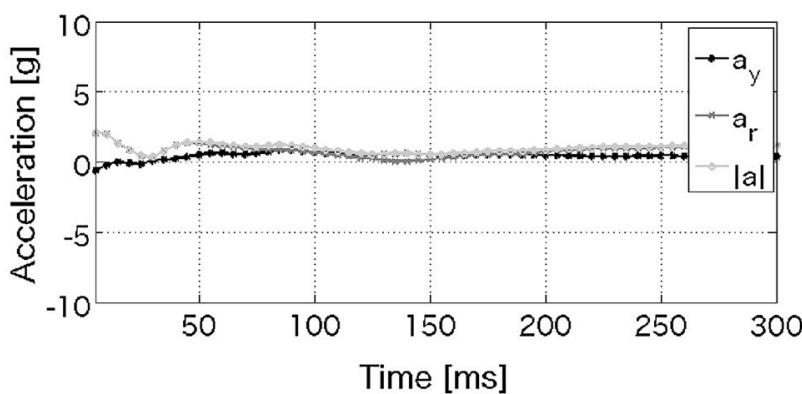

d

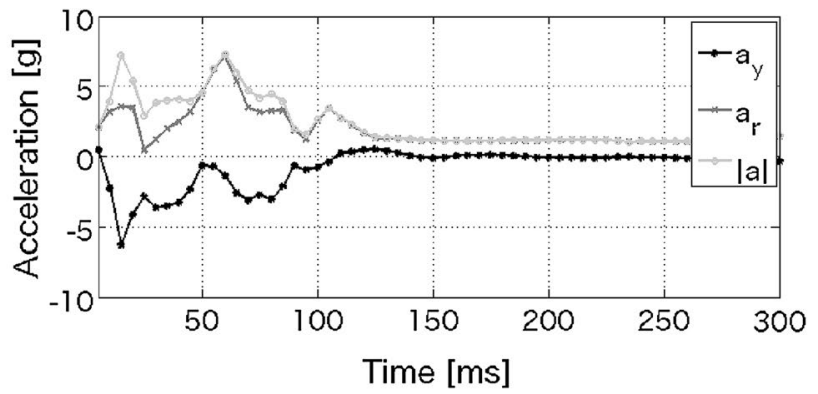

e

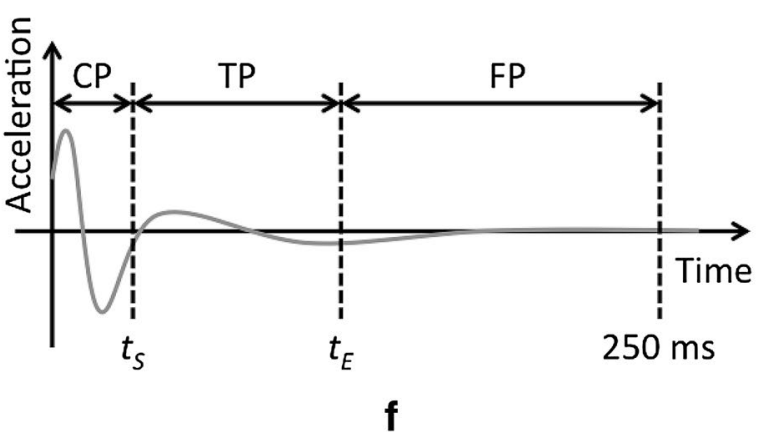

Fig. 2 A typical example of changes in three-axis acceleration associated with a shrimp-eating, b fish-eating, $\mathbf{c}$ crab-eating, $\mathbf{d}$ others (routine) and e others (escape). $\mathbf{f}$ A schematic diagram of three phases, i.e., commencement phase (CP), transition phase (TP) and final phase (FP), demarcated by border times $t_{S}$ and $t_{E}$. The horizontal axis shows the time elapsed from the trigger point, and the vertical axis acceleration 


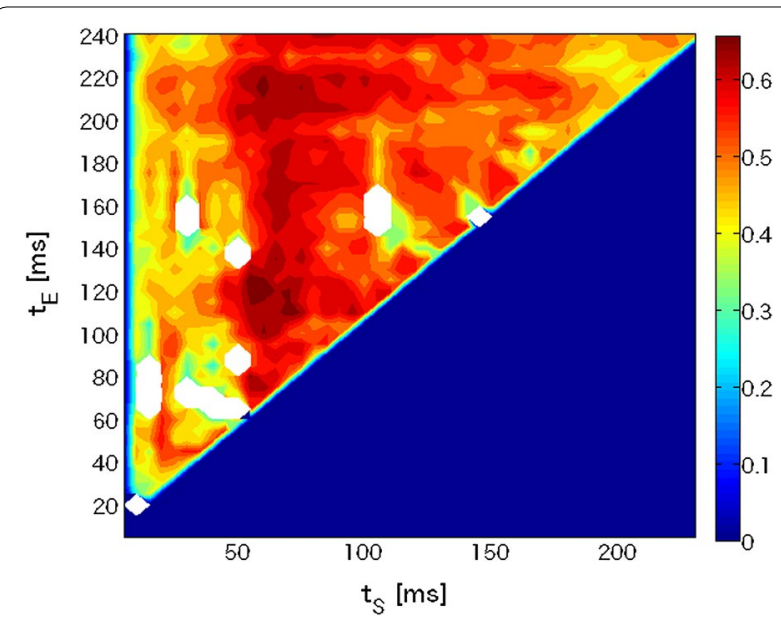

Fig. 3 A contour map identifying the optimum border times $t_{5}$ and $t_{E}$. The horizontal, vertical and colored axes show border time $1\left(t_{S}\right)$, border time $2\left(t_{E}\right)$ and the harmonic mean of the $F$-measure for all types of behaviors, respectively

probability density of each behavioral pattern in each cell was calculated. Each cell was labeled with the behavioral pattern for which the probability density was greatest. We thus obtained the 3D lookup table shown in Fig. 6.

We ran a classification test using fivefold cross-validation (Table 2). Of all behaviors, 38/49 (78\%) shrimp-eating, 6/9 (67\%) fish-eating, 10/15 (67\%) crab-eating and 25/45 (56\%) other behaviors were correctly classified. However, many shrimp-eating and other behaviors were misclassified as crab-eating. This was because some aspects of crabeating were very similar to those of shrimp-eating and other behaviors. Therefore, we chose additional parameters to discriminate among shrimp-eating, crab-eating and other behaviors classified as crab-eating.

\section{Classification phase II}

The strongest candidate for parameter IV was the $a_{r}$ of the early portion of behaviors; acceleration that was orthogonal to the body axis was rather independent of parameter I. We calculated $p$ values between (a) shrimp-eating and crab-eating, (b) crab-eating and other behaviors and (c) other behaviors and shrimp-eating, as functions of the border times (the times from event initiation) (Fig. 7a). We found that other behaviors could be reasonably well separated $(p<0.01)$ when the border time was $15 \mathrm{~ms}$. We set the threshold where the Mahalanobis distances (a statistical distance from the mean [18]) of crab-eating and other behaviors were equal (Fig. 7b).

Figure $7 \mathrm{a}$ shows that the $p$ value comparison between shrimp-eating and crab-eating decreased as the border time exceeded $70 \mathrm{~ms}$. This indicated that the later part of $a_{r}$ might serve as parameter $V$, which could distinguish shrimp-eating from crab-eating. We calculated $p$ values as functions of the time to the end of an event $(250 \mathrm{~ms})$ (Fig. 8a). We found that the $p$ value for the comparison between shrimp-eating and crab-eating reached its lowest level $(p<0.01)$ when the border time was $140 \mathrm{~ms}$. We thus set this as the threshold so that true-positive crabeating events were not discarded (Fig. 8b).

Finally, we fixed the classification flow as described in Fig. 9. The classification test results were revised as shown in Table 3. Compared to Table 2, eight events of other behavior and four events of shrimp-eating were

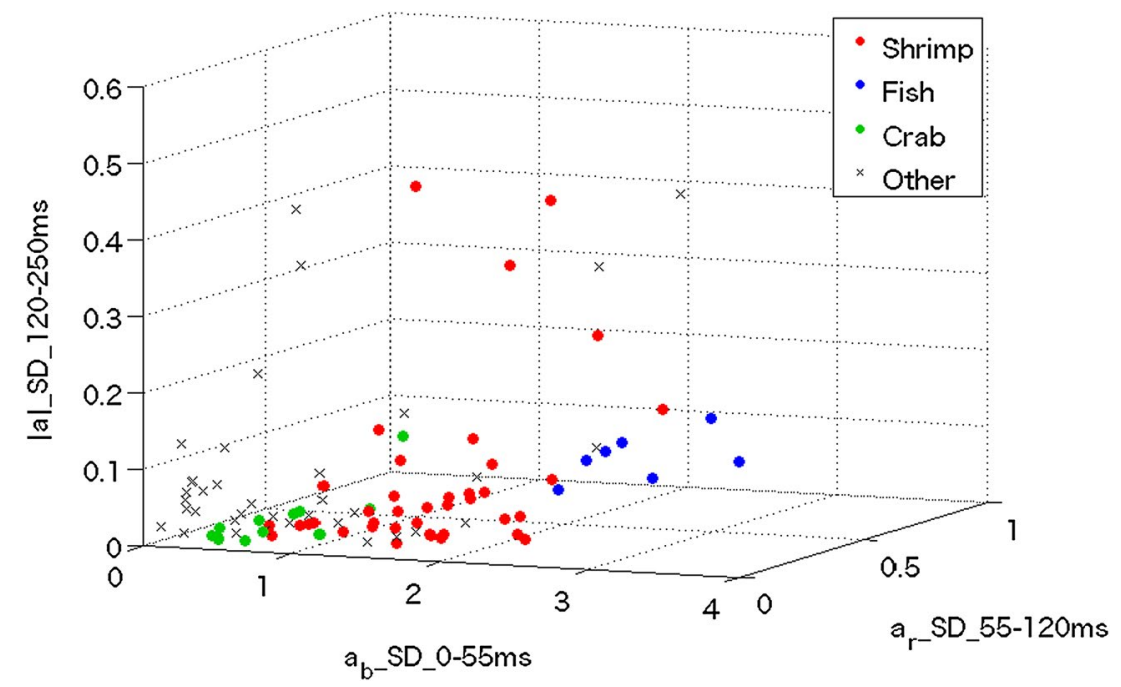

Fig. 4 Scatter plots of all behaviors. Each circle is plotted at a location specified by three parameters. The terms 'a $a_{b-} S D_{-} \_-55$ ms,' $a_{r-} S D \_55-120$ ms' and '|a|_SD_120-250 ms' are the standard deviations of body-axis acceleration from 0 to $55 \mathrm{~ms}$, the standard deviation of orthogonal acceleration from 55 to $120 \mathrm{~ms}$ and the standard deviation of the length of the acceleration vector from 120 to $250 \mathrm{~ms}$, respectively 

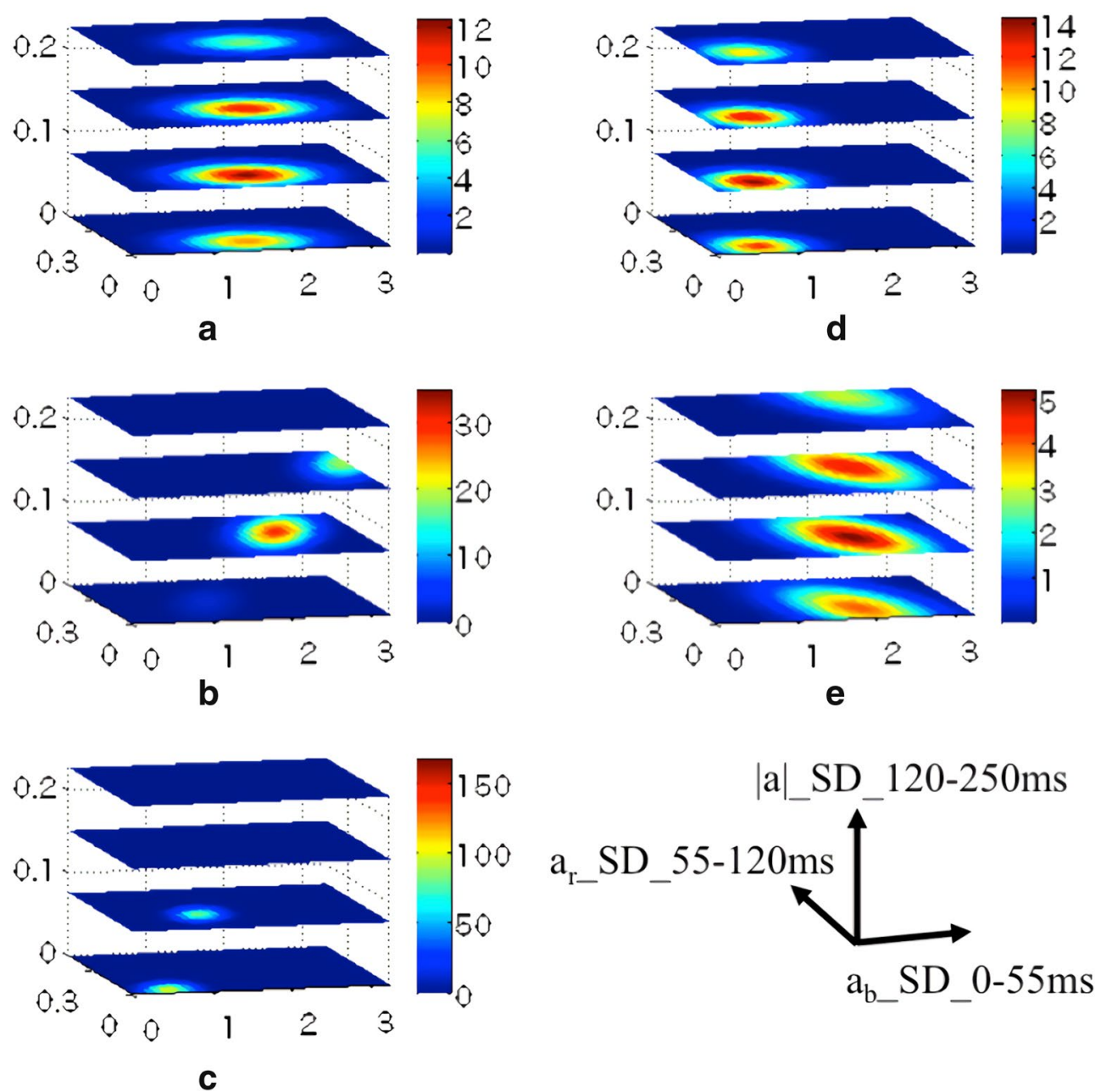

Fig. 5 Probability densities of shrimp-eating, fish-eating, others (routine) and others (escape). The probability of each type of behavior is high in the red zones and low in the blue zones. a Shrimp-eating, b fish-eating, c crab-eating, $\mathbf{d}$ others (routine), e others (escape)

successfully eliminated from the totals of crab-eating. Finally, the $F$-measures, the harmonic means (thus balanced indicators) of precision and recall, came down to $0.77 \pm 0.11$ for shrimp-eating, $0.73 \pm 0.19$ for fisheating, $0.71 \pm 0.22$ for crab-eating and $0.78 \pm 0.04$ for other behaviors (the figures are means with standard deviations).

\section{Discussion}

We found that the feeding behaviors of red-spotted groupers could be detected and classified to accuracies of 0.77 for shrimp-eating, 0.73 for fish-eating and 0.71 for crab-eating in the $F$-measures, employing only acceleration data along the body axis and orthogonal direction. These accuracies were somewhat less but still comparable to those produced by employing both acceleration and angular velocity [4]. We selected five parameters, i.e., the standard deviation of $a_{b}$ of 0-55 ms, the standard deviation of $a_{r}$ of 55-120 ms, the standard deviation of $|a|$ of $120-250 \mathrm{~ms}$, the standard deviation of $a_{r}$ of $0-15 \mathrm{~ms}$ and the standard deviation of $a_{r}$ of $140-250 \mathrm{~ms}$, for the classification algorithm. The behavioral patterns of red-spotted groupers are associated with fairly specific amplitudes and durations of acceleration. Thus, we could classify feeding behaviors accurately using time-specific parameters derived from only acceleration data.

Although target fish need to be within the range of a receiver (several hundred meters for example; cf. AQRM1000, AquaSound Inc., Kobe, Japan) when data are transmitted, it would be possible to count and log the number of each behavior in the memory of a pinger. These data could then be transmitted to the receiver when the fish is 


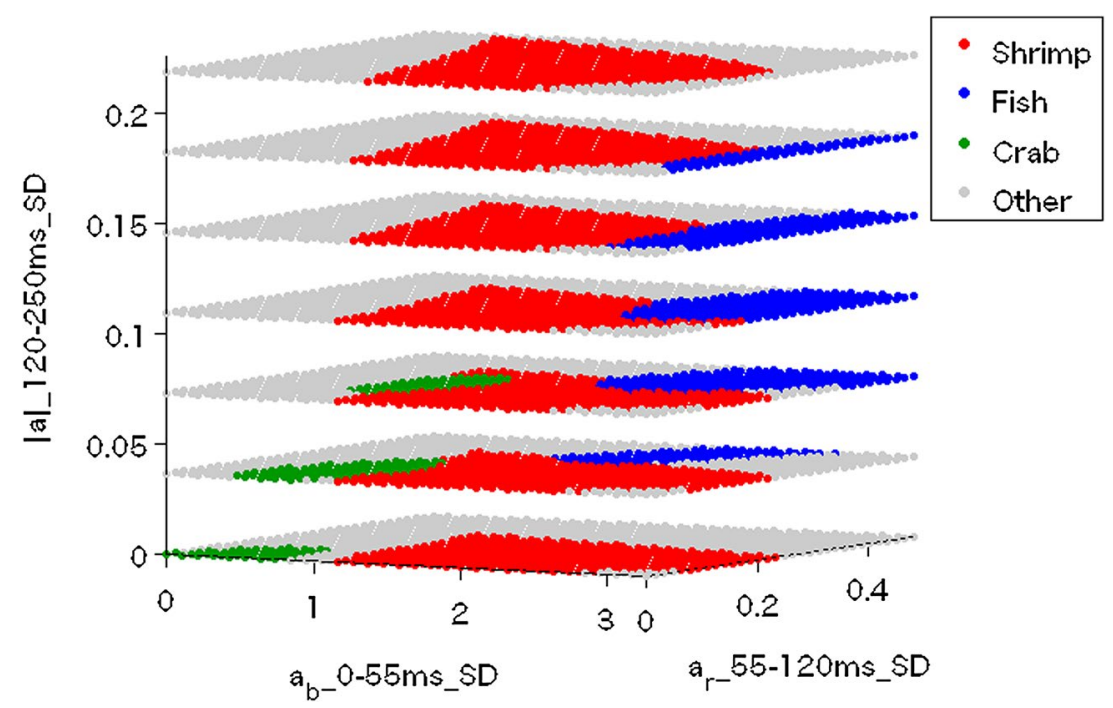

Fig. 6 Three-dimensional lookup table that determines behavioral types. The most probable behavioral pattern at each point is assigned. For example, if the acceleration data parameters are 2, 0.5 and 0.1 , respectively, the behavior is classified as shrimp-eating

Table 2 Classification test results using the lookup table

\begin{tabular}{lllcc}
\hline True events & \multicolumn{3}{l}{ Classified as } & \\
\cline { 2 - 5 } & Shrimp & Fish & Crab & Other \\
\hline Shrimp & 38 & 2 & 6 & 3 \\
Fish & 1 & 6 & 0 & 2 \\
Crab & 4 & 0 & 10 & 1 \\
Other & 11 & 0 & 9 & 25 \\
\hline
\end{tabular}

within its detection range. Therefore, the feeding behaviors of a target fish do not always need to be performed within the detection range of the receiver.

Many previous studies assessing the feeding behavior of fish utilized data loggers. A data logger can measure a comparatively wide range of variables, such as acceleration, angular velocity and magnetic parameters at high frequency. Such variables have enabled us to accurately

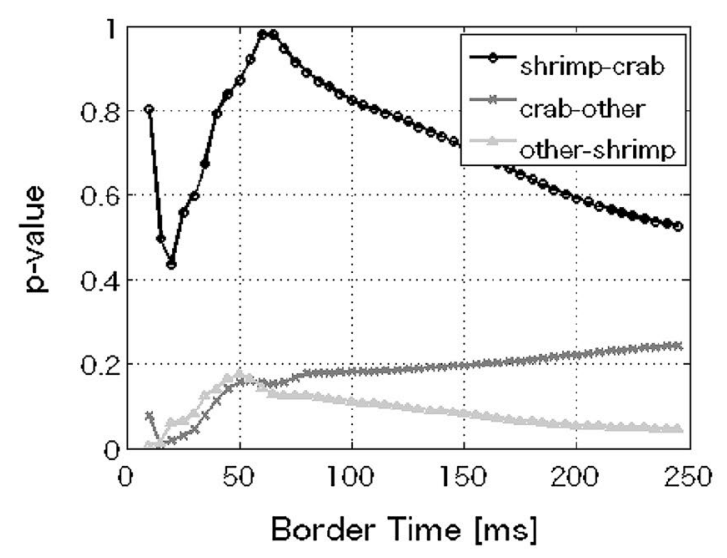

a

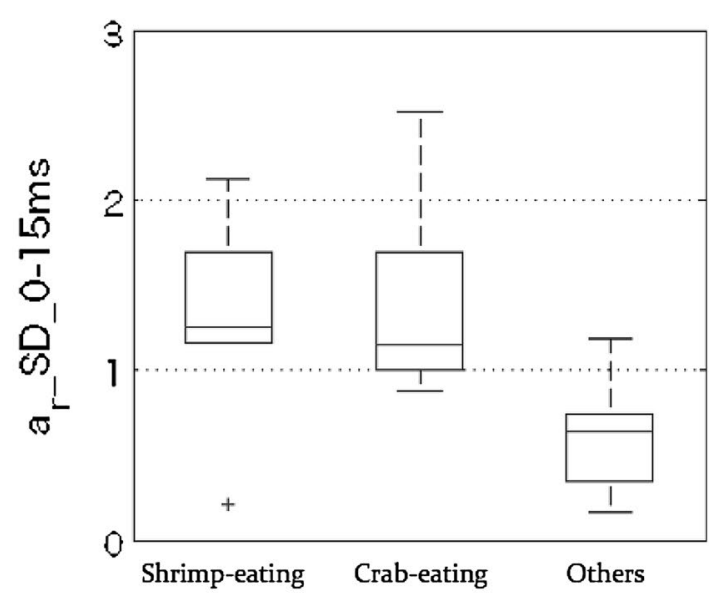

b

Fig. 7 a $p$ values by the border time from event initiation. The $p$ value between crab-eating and other behaviors was minimal when the border time was $0.15 \mathrm{~ms}$. b A box plot of the parameter ar_SD_0-15 ms, which describes the standard deviation of orthogonal acceleration from 0 to $15 \mathrm{~ms}$. Events in which this parameter was less than 0.84 were classified as other behaviors 


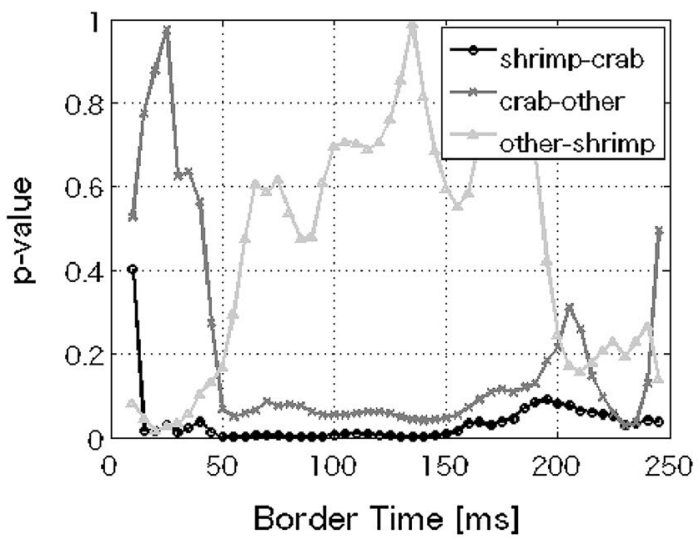

a

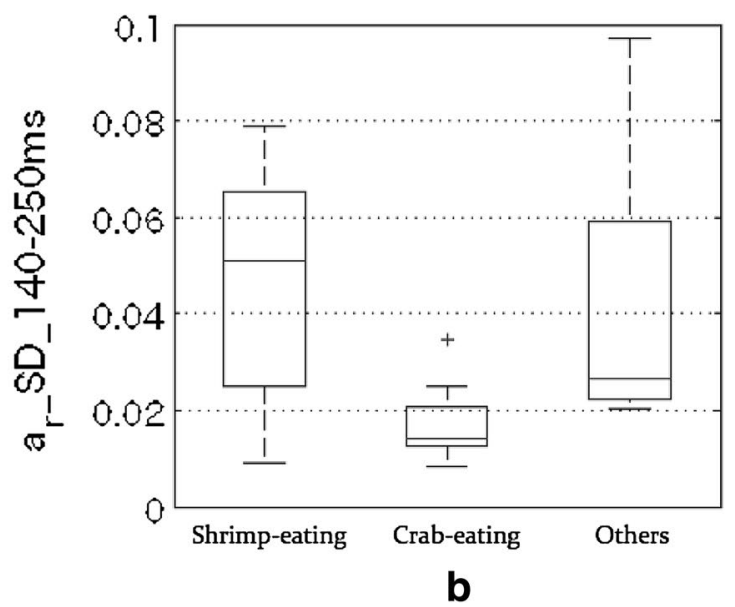

b

Fig. 8 a $p$ value versus the border time to the end of the event. The $p$ value between crab-eating and shrimp-eating was minimal when the border time was $140 \mathrm{~ms}$. b A box plot of the parameter ar_SD_140-250 ms, which describes the standard deviation of orthogonal acceleration from 140 to $250 \mathrm{~ms}$. Events for which this parameter was less than 0.04 were classified as crab-eating

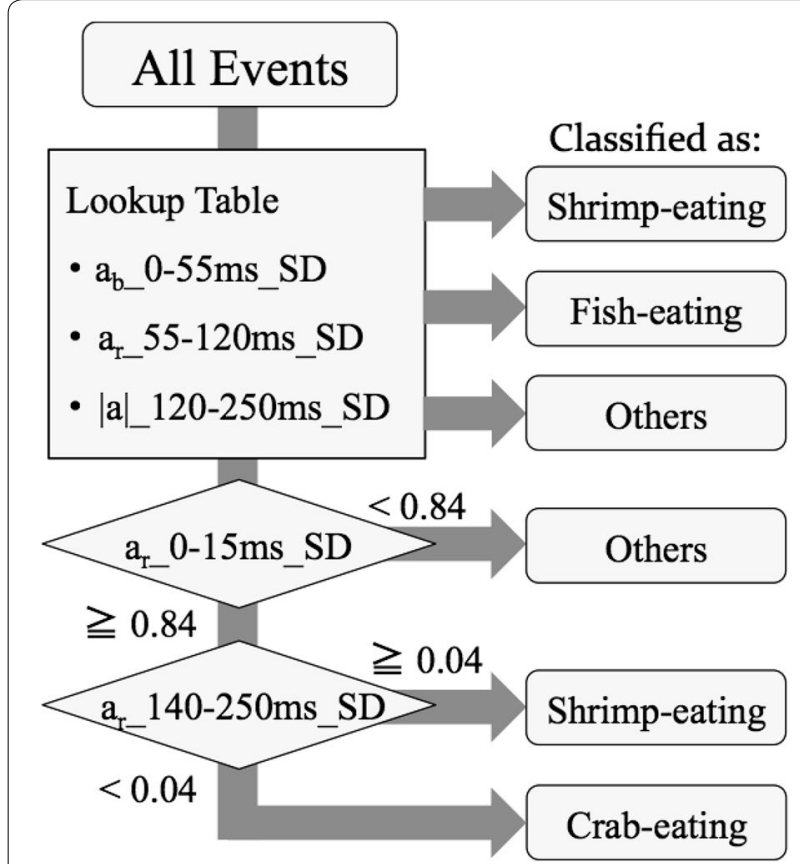

Fig. 9 Flow of classification. The first fork presents the three-dimensional lookup table and the other forks illustrate the decision tree

detect and identify specific behaviors, including feeding behaviors, of fish [4, 19]. However, a data logger must be retrieved after being attached to a target animal to download data. On the other hand, a pinger can transmit data remotely, while the amount of data it can transmit are technically limited [13]. Previous studies have succeeded in assessing the field activity of fish using a V9AP (VEMCO Inc., Nova Scotia, Canada), along with several specific behaviors of fish such as burrowing movements and tail beat activity using AccelTag (Thelma Biotel, Trondheim, Norway). However, remote assessment of feeding behavior in fish has remained challenging.

In this study, we developed an algorithm for a new acceleration pinger that classifies the feeding behavior of fish. The algorithm will be incorporated in the pinger, which records acceleration data at high frequency $(200 \mathrm{~Hz})$, processes the data onboard and outputs classification results of behaviors. We used red-spotted groupers as a test species and showed that their feeding behaviors could be classified with accuracies of more than 0.7 (in the $F$-measures) using acceleration data. However, the behavior of red-spotted groupers in experimental tanks will differ somewhat from that in the wild. The frequency of each type of behavior could vary with the surrounding environment. In addition, each fish will interact with other individuals, creating other movements in addition to the routine and escape movements. Thus, the accuracy of classification may be reduced to some extent in the field. Nevertheless, our method could be applied to any animal of sufficient size to accommodate the experimental instrument by adapting the classification algorithm, although it might be fairly difficult to apply this method to fish that do not employ ambush hunting strategies, as these species may be more likely to move completely out of the receiver's range and may not make striking acceleration when feeding. The results of previous studies support the applicability of our method (e.g., $[3,4])$. The method by which this procedure could be adapted to use in other species would be as follows: (1) conducting tank experiments using acceleration data loggers and a video camera, (2) analyzing acceleration data 
Table 3 Classification test results using both the lookup table and the decision tree

\begin{tabular}{lllcc}
\hline True events & \multicolumn{3}{l}{ Classified as } & \\
\cline { 2 - 5 } & Shrimp & Fish & Crab & Other \\
\hline Shrimp & 41 & 2 & 2 & 4 \\
Fish & 1 & 6 & 0 & 2 \\
Crab & 4 & 0 & 10 & 1 \\
Other & 11 & 0 & 1 & 33 \\
\hline
\end{tabular}

to find the motions common to each behavioral pattern, (3) choosing several relevant parameters to construct a classification algorithm (either a lookup table or a decision tree) and (4) programming the algorithm into an acceleration transmitter. This method could also potentially be applied to animals other than fish. For example, the feeding strategies of seabirds and marine mammals are also of interest to biologists (cf research using acceleration data loggers and/or ARGOS transmitters [20-22] and a review paper [23]), but research in this area has been hindered by problems such as the difficulty of tag retrieval and low frequencies of data transmission. Methods for remote sensing of accelerational events in such animals could also be broadened using this method.

\section{Conclusions}

Feeding is one of the most fundamental elements of fish biology, as are breeding and death [15]. The feeding behavior of fish critically affects growth and maturation and plays an important role in estimating the environmental accommodations of their habitats. However, methods used to assess the feeding behavior of fish in the field have mainly been limited to enumerating stomach contents $[15,24,25]$ and video-recording [26]. We developed a new method, using an acceleration pinger, to assess the feeding behaviors of fish in the wild and showed that it may be possible to use this method to investigate other aspects of fish biology, such as individual feeding strategies.

\section{Abbreviations}

RMS: root mean square (statistical measure of the magnitude of varying quantity); TL: total length; BM: body mass; FRP: fiber-reinforced plastic.

\section{Authors' contributions \\ $J \mathrm{H}, \mathrm{HM}$ and $\mathrm{TS}$ designed and developed the acceleration pinger. JH, $\mathrm{Yl}$ and $\mathrm{YM}$ conducted tank experiments using acceleration data loggers. JH, YI, YM and TN analyzed the acceleration data. JH, HM, TN, KM and NA wrote the draft of manuscript. All authors read and approved the final manuscript.}

\section{Author details}

${ }^{1}$ Graduate School of Informatics, Kyoto University, Yoshida-honmachi, Sakyo-ku, Kyoto, Kyoto 606-8501, Japan. ${ }^{2}$ AquaSound Inc., Portisland Building 501, 4-1-1 Minato-jima Nakamachi, Chuo-ku, Kobe, Hyogo 650-0046, Japan.
${ }^{3}$ CREST, Japan Science and Technology Agency, Kawaguchi Center Building, 4-1-8 Hon-cho, Kawaguchi, Saitama 332-0012, Japan. ${ }^{4}$ The Institute of Statistical Mathematics, 10-3 Midori-cho, Tachikawa, Tokyo 190-8562, Japan. ${ }^{5}$ Field Science Education and Research Center, Kyoto University, Kitashirakawa Oiwake-cho, Sakyo-ku, Kyoto, Kyoto 606-8502, Japan. ${ }^{6}$ Present Address: Graduate School of Informatics, Kyoto University, Yoshida-honmachi, Sakyo-ku, Kyoto, Kyoto 606-8501, Japan.

\section{Acknowledgements}

We thank all the members of Maizuru Fisheries Research Station for all the support on the tank experiments. We sincerely acknowledge Katsumi Ito and Yasuhiko Endo (AquaSound Inc.) for all the helps and advices with respect to the acceleration pinger.

\section{Competing interests}

The authors declare that they have no competing interests.

\section{Ethics approval and consent to participate}

All procedures of the experiments were approved by the Animal Research Committee of Kyoto University (Permit Numbers: Informatics 25-3 and Inf-K14003).

\section{Availability of data and materials}

The datasets used and/or analyzed during the current study available from the corresponding author on reasonable request.

\section{Funding}

The funding for this study was obtained from Grant-in-Aid for Young Scientists (A) (25712022).

\section{Publisher's Note}

Springer Nature remains neutral with regard to jurisdictional claims in published maps and institutional affiliations.

Received: 18 December 2016 Accepted: 18 May 2017

Published online: 08 June 2017

\section{References}

1. Domenici P, Blake R. The kinematics and performance of fish fast-start swimming. J Exp Biol. 1997;200(8):1165-78.

2. Broell F, Noda T, Wright S, Domenici P, Steffensen JF, Auclair JP, Taggart CT. Accelerometer tags: detecting and identifying activities in fish and the effect of sampling frequency. J Exp Biol. 2013;216:1255-64.

3. Noda T, Kawabata Y, Arai N, Mitamura H, Watanabe S. Monitoring escape and feeding behaviours of cruiser fish by inertial and magnetic sensors. PLOS ONE. 2013:8:e79392.

4. Kawabata Y, Noda T, Nakashima Y, Nanami A, Sato T, Takebe T, Soyano K. Use of a gyroscope/accelerometer data logger to identify alternative feeding behaviours in fish. J Exp Biol. 2014;217(18):3204-8.

5. Cooke SJ, Hinch SG, Wikelski M, Andrews RD, Kuchel L, Wolcott TG, Butler PJ. Biotelemetry: a mechanistic approach to ecology. Trends Ecol Evol. 2004;19:334-43.

6. Murchie KJ, Cooke SJ, Danylchukc AJ, Suski CD. Estimates of field activity and metabolic rates of bonefish (Albula vulpes) in coastal marine habitats using acoustic tri-axial accelerometer transmitters and intermittent-flow respirometry. J Exp Mar Biol Ecol. 2011;396:147-55.

7. Payne NL, Gillanders BM, Seymour RS, Webber DM, Snelling EP, Semmens JM. Accelerometry estimates field metabolic rate in giant Australian cuttlefish Sepia apama during breeding. J Anim Ecol. 2011;80:422-30.

8. O'Toole AC, Murchie KJ, Pullen C, Hanson KC, Suski CD, Danylchuk AJ, Cooke SJ. Locomotory activity and depth distribution of adult great barracuda (Sphyraena barracuda) in Bahamian coastal habitats determined using acceleration and pressure biotelemetry transmitters. Mar Freshw Res. 2010;61:1446-56.

9. Kneebone J, Chisholm J, Skomal G. Mar Biol. 2014;160(5):1149-63. 
10. Wilson SM, Hinch SG, Eliason EJ, Farrell AP, Cooke SJ. Calibrating acoustic acceleration transmitters for estimating energy use by wild adult Pacific salmon. Comp Biochem Physiol A. 2013;164:491-8.

11. Watson WH III, Johnson SK, Whitworth CD, Chabot CC. Rhythms of locomotion and seasonal changes in activity expressed by horseshoe crabs in their natural habitat. Mar Ecol Prog Ser. 2016;542:109-21.

12. Føre M, Alfredsen JA, Gronningsater A. Development of two telemetrybased systems for monitoring the feeding behaviour of Atlantic salmon (Salmo salar L.) in aquaculture sea-cages. Comput Electron Agric. 2011;76(2):240-51.

13. Almeida PR, Pereira TJ, Quintella BR, Gronningsaeter A, Costa MJ, Costa $J$ L. Testing a 3-axis accelerometer acoustic transmitter (AccelTag) on the Lusitanian toadfish. J Exp Mar Biol Ecol. 2013;449:230-8.

14. Yoshida N, Mitamura H, Sasaki M, Nobuaki N. Measurement of activity for sit-and-wait predator, red-spotted grouper, using acoustic acceleration transmitter. J Adv Mar Sci Technol Soc. 2015;21(1):1-5.

15. Kayano Y. Feeding Habits of the Grouper, Epinephelus akaara, on Artificial Reefs. Suisanzoshoku. 2001;49(1):15-21.

16. Ukawa M, Higuchi M, Mito S. Spawning habits and early life history of a serranid fish, Epinephelus akaara. Jpn J Ichthyol. 1966;13:156-61.

17. Masuda R, Matsuda K, Masaru T. Laboratory video recordings and underwater visual observations combined to reveal activity rhythm of red-spotted grouper and banded wrasse, and their natural assemblages. Environ Biol Fish. 2012;95:335-46.

18. Mahalanobis PC. On the generalized distance in statistics. Proc Natl Inst Sci India. 1936;12:49-55.
19. Noda T, Kawabata Y, Arai N, Mitamura H, Watanabe S. Animal-mounted gyroscope/accelerometer/magnetometer: in situ measurement of the movement performance of fast-start behaviour in fish. J Exp Mar Biol Ecol. 2013;451:55-68.

20. Gallon S, Bailleul F, Charrassin J-B, Guinet C, Handrich Y, Hindell M. Identifying foraging events in deep diving southern elephant seals, Mirounga leonina, using acceleration data loggers. Deep-Sea Res. 2013;88-89:14-22.

21. Naito Y, Bornemann $H$, Takahashi A, Mclntyre T, Plotz J. Fine-scale feeding behavior of Weddell seals revealed by a mandible accelerometer. Polar Sci. 2010;4:309-16.

22. Naito Y, Costa DP, Adachi T, Robinson PW, Fowler M, Takahashi A. Unravelling the mysteries of a mesopelagic diet: a large apex predator specializes on small prey. Funct Ecol. 2013;27:710-7.

23. Wilson RP, Vandenabeele SP. Technological innovation in archival tags used in seabird research. Mar Ecol Prog Ser. 2012;451:245-62.

24. Hyslop EJ. Stomach contents analysis - a review of methods and their application. J Fish Biol. 1980;17(4):411-29.

25. Cortés E. A critical review of methods of studying fish feeding based on analysis of stomach contents: application to elasmobranch fishes. Can J Fish Aquat Sci. 1997;54(3):726-38.

26. Hooker SK, Boyd IL, Jessopp M, Cox O, Blackwell J, Boveng PL, Bengtson $J$ L. Monitoring the prey-field of marine predators: combining digital imaging with datalogging tags. Mar Mamm Sci. 2002;18(3):680-97.

\section{Submit your next manuscript to BioMed Central and we will help you at every step:}

- We accept pre-submission inquiries

- Our selector tool helps you to find the most relevant journal

- We provide round the clock customer support

- Convenient online submission

- Thorough peer review

- Inclusion in PubMed and all major indexing services

- Maximum visibility for your research

Submit your manuscript at www.biomedcentral.com/submit 